\title{
UN ABORDAJE COMPARATIVO EN TORNO A LA INCIDENCIA DE LA TUBERCULOSIS EN INMIGRANTES BOLIVIANOS DE BUENOS AIRES Y SÃO PAULO
}

\author{
Alejandro Goldberg*
}

\begin{abstract}
Este trabajo expone de manera exploratoria y desde una perspectiva sociosanitaria regional, en clave comparativa, algunas dimensiones de análisis en torno a la incidencia de la tuberculosis en inmigrantes bolivianos de las ciudades de Buenos Aires y São Paulo. En ambos casos, se trata de un padecimiento ligado a sus modos de vida y de trabajo precarios en talleres textiles clandestinos. Sumado a lo anterior, la falta de documentación en regla, las dificultades de acceso a la atención sanitaria pública y el sufrimiento social experimentado, potencian la situación de vulnerabilidad social de estas personas; aumentando los riesgos de infectarse y desarrollar la enfermedad.
\end{abstract}

Palabras clave: Inmigrantes bolivianos; Talleres textiles clandestinos; Vulnerabilidad social; Tuberculosis.

\section{Introducción}

Dentro del sistema migratorio actual del Cono Sur, tanto Argentina como Brasil se caracterizarían por constituir históricamente países de atracción y recepción de migrantes limítrofes, tal como lo fueron entre finales del siglo XIX y principios del XX de inmigrantes transatlánticos. ${ }^{1}$ Del lado opuesto, países como Paraguay, Bolivia y Perú, han sido, sobre todo con posterioridad a la segunda mitad del siglo XX, fundamentalmente "países emisores" de mano de obra.

\footnotetext{
"Doctor y Máster en Antropología de la Medicina (Universidad Rovira y Virgili, España). Licenciado en Ciencias Antropológicas por la Universidad de Buenos Aires. Investigador Adjunto del Consejo Nacional de Investigaciones Científicas y Técnicas de la República Argentina en el Instituto de Ciencias Antropológicas, Sección de Antropología Social (FFyL-UBA). Profesor del Seminario Antropología y Migraciones Internacionales (UBA). E-mail: alejandro.goldberg@gmail.com. Buenos Aires/Argentina.

1 BALÁN, Jorge. La economía doméstica y las diferencias entre los sexos en las migraciones internacionales: un estudio sobre el caso de los bolivianos en la Argentina.
} 
En el marco de los flujos migratorios que se producen desde la década de 1990 en adelante, como parte del proceso globalizador, Buenos Aires y São Paulo (de ahora en adelante BA y SP, respectivamente), incluidas sus áreas metropolitanas, se convierten en destino de una joven, creciente y variada en términos de género y origen - migración boliviana. ${ }^{2}$

Los procesos de inserción sociolaboral, así como aquellos vinculados con la integración de estos inmigrantes a los contextos de estas dos urbes, estuvieron marcados desde sus inicios por distintos tipos de conflictos, estigmas y construcciones sociales discriminatorias de parte de sectores locales de ambas sociedades. Una de las razones de ello radica en que dichos procesos se desarrollaron en situaciones de mayor precariedad (social, económica, laboral, habitacional, etc.) que aquellas con las que se encontraron, en una época muy distinta, casi un siglo atrás los inmigrantes europeos. ${ }^{3}$

En el caso específico de los bolivianos, estos constituyen el mayor grupo de inmigrantes limítrofes recientes en SP (el segundo en BA, detrás de los paraguayos). Al respecto, tres son, al menos, las características generales compartidas por este colectivo inmigrante en relación a su presencia en las dos ciudades $\left.^{4}: 1\right)$ predominancia de su condición de clandestinidad (irregularidad administrativa-migratoria); 2) están sometidos a un importante proceso de estigmatización por parte de sectores de la población local (mecanismos de estereotipación fenotípica y cultural); y 3) se insertan en el ámbito sumergido de la economía, ocupando puestos de trabajo precarizados que en algunos casos rozan las condiciones de esclavitud, ${ }^{5}$ como analizaremos a continuación al abordar sus modos de vida/trabajo/vivienda en los talleres textiles clandestinos (de ahora en adelante TTC); y las situaciones de riesgo para la salud que estos suponen, focalizando en la incidencia de la tuberculosis (de ahora en adelante TBC), su contagio y transmisión, entre los inmigrantes bolivianos y sus familias.

${ }^{2}$ Los últimos datos proporcionados por la Organización Internacional para las Migraciones (OIM) muestra que en la última década, de los 700.000 sudamericanos que salieron de sus países, 500.000 llegaron a la Argentina, encabezados por paraguayos y bolivianos. El segundo destino para estos migrantes fue Brasil.

${ }^{3}$ GOLDBERG, Alejandro; SILVEIRA, Cássio. Modos de vida, condiciones de acceso a la salud pública y procesos de atención en inmigrantes bolivianos de Buenos Aires y São Paulo: una indagación comparativa.

${ }^{4}$ Para un análisis sobre las diferencias y especificidades en la evolución del proceso migratorio boliviano en Brasil/São Paulo y Argentina/Buenos Aires, pueden consultarse FRACASSO TAMBELLINI, Elaine et alii. Inclusão de imigrantes bolivianos na cidade de São Paulo: algumas estratégias e políticas locais; SOUCHAUD, Sylvain; BAENINGER, Rosana. Collas e cambas do outro lado da fronteira: aspectos da distribuição diferenciada da imigração boliviana em Corumbá, Mato Grosso do Sul, para el caso brasilero; mientras que para el caso argentino, SASSONE, Susana; DE MARCO, Graciela. Problemáticas territoriales de la inmigración limítrofe; GRIMSON, Alejandro. Relatos de la diferencia y la igualdad. Los bolivianos en Buenos Aires; BENENCIA, Roberto. Apéndice: La inmigración limítrofe.

${ }^{5}$ GOLDBERG, SILVEIRA, op. cit., p. 17. 


\section{Incorporación de mano de obra boliviana irregular en el ámbito sumergido y precario de la industria textil de São Paulo y Buenos Aires}

En trabajos anteriores he abordado el proceso migratorio de una parte de la migración boliviana actual a BA y su área metropolitana, describiendo en particular el procedimiento de incorporación ilegal de inmigrantes bolivianos al sector sumergido de la industria textil. ${ }^{6}$ Contrariamente a lo que sucede al compararlo con la dinámica general de otros procesos migratorios, en el caso analizado son una parte de las redes y cadenas migratorias, creadas y reproducidas por miembros de la colectividad boliviana (familiares, parientes, amigos, vecinos, paisanos, etc.), las que articulan mecanismos de tráfico, trata y reducción a la servidumbre de adultos y menores, utilizadas en un doble sentido: tanto para la captación de trabajadores en origen - la mayor de las veces engañados - como para la sujeción posterior de estos - en muchos casos con sus hijos - a los TTC. Del mismo modo, estarían operando mecanismos de trata y tráfico de personas para el reclutamiento y la reducción a la servidumbre de inmigrantes bolivianos en talleres ("oficinas") de costura de SP.

En este sentido, aun reconociendo las especificidades de cada contexto y las particularidades de cada proceso, resultan reveladoras para los fines del análisis que aquí se propone las semejanzas encontradas al indagar en el caso de los inmigrantes bolivianos de la ciudad de SP. ${ }^{8}$ Lo anterior, no sólo en términos cuantitativos, como se mencionó anteriormente; sino, sobre todo, porque, en buena medida, estos se insertan en el ámbito sumergido de la economía, principalmente en el sector textil y en una condiciones de precariedad laboralhabitacional que los coloca en una grave situación de vulnerabilidad social: “(...) a imigração dos bolivianos para São Paulo, mais do que uma questão de pobreza na Bolívia, está ligada, sobretudo a uma forma de desenvolvimento econômico de uma divisão da industria de confecções paulistana". ${ }^{9}$

Las similitudes entre los dos casos expuestos tienen su correspondencia también al momento de identificar la forma en que los empresarios locales (brasileros y coreanos en SP; argentinos y coreanos en BA), para evadir

\footnotetext{
${ }^{6}$ GOLDBERG, Alejandro. Tuberculosis y vulnerabilidad social. Una investigación etnográfica sobre inmigrantes bolivianos que trabajan y viven en talleres textiles clandestinos del Área Metropolitana de Buenos Aires; IDEM. Servitude and slave trade: the case of Bolivian immigrants who work in clandestine textile workshops of the Buenos Aires Metropolitan Area; IDEM. Análisis de la relevancia de los factores socioculturales en el proceso asistencial de pacientes con tuberculosis, usuarios del Instituto VaccarezzaHospital Muñiz. Un abordaje etnográfico desde la Antropología Médica, entre otros.

${ }^{7}$ DA SILVA, Sidney. Bolivianos em São Paulo: entre o sonho e a realidade.

${ }^{8}$ Para explorar en profundidad las particularidades del caso paulista, puede consultarse el trabajo de BUECHLER, Simone. Sweating It in the Brazilian Garment Industry: Korean and Bolivian Immigrants and Global Economic Forces in São Paulo.

${ }^{9}$ FREIRE DA SILVA, Carlos. Precisa-se: bolivianos na industria de confecções em São Paulo, p. 10.
} 
controles y maximizar la ganancia, tercerizan la producción en talleres regenteados por bolivianos, ex trabajadores semi esclavos en TTC que en algún momento pudieron regularizar su situación migratoria. De modo que en la industria de confección de indumentaria, la descentralización y tercerización de la producción para rebajar costos y maximizar la ganancia es lo que conlleva a los talleres a producir en esas condiciones de clandestinidad y de sobreexplotación de la mano de obra: en el caso argentino, por una prenda que en el mercado se vende a $\$ 100$, el trabajador recibe como paga $\$ 1,8$, mientras que al dueño del taller le corresponde \$1,30. El resto se distribuye de la siguiente manera: el 64\% queda en el circuito de comercialización, un $11 \%$ representa el costo de las materias primas y el $22 \%$ corresponde a los impuestos. Por otro lado, se estima que el $80 \%$ de la confección de prendas tercerizadas en talleres se realiza bajo condiciones informales o violando los derechos humanos básicos. ${ }^{10}$

El mecanismo de explotación intracolectivo en el sector constituye otro de los rasgos en común identificados, reproduciéndose entre los mismos bolivianos en las dos ciudades: aquel que está hace más tiempo, consiguió su documentación y fue explotado previamente, es contratado para reclutar, traer y explotar al compatriota recién llegado, que se encuentra en condiciones más vulnerables que él. Muchos inmigrantes bolivianos que comienzan a trabajar en TTC del Área Metropolitana de Sao Paulo (AMSP) lo viven como una fase transitoria, un sacrificio momentáneo que tiene un determinado tiempo de duración para conseguir un ascenso social; el cual implicaría regularizar su situación migratoria, comprarse una máquina de coser, montarse su propio taller textil clandestino y explotar a otros trabajadores en situación de mayor vulnerabilidad (es decir, en una fase previa como la que experimentó él mismo al comenzar). ${ }^{11}$ En el caso de BA, los testimonios recogidos en el transcurso del trabajo de campo etnográfico realizado muestran que en el momento de contratarlos en Bolivia, los agentes locales de los talleristas bolivianos hacen copias de los documentos de todos los integrantes de la familia del trabajador. De esta manera, una vez que se instalan en los talleres, los dueños amenazan a los trabajadores diciéndoles, por un lado, que si se rebelan, escapan o accionan en contra de sus intereses, los familiares que quedaron en Bolivia "pagarán" las consecuencias. Por otro lado, frecuentemente la amenaza consiste en llamar a la policía: a pesar de lo paradójico que parezca el caso, en el cual el mismo responsable de una actividad ilegal (violación de la ley 12.713 de trabajo a domicilio en Argentina) puede hacer las veces

\footnotetext{
${ }^{10}$ LIEUTIER, Ariel. Esclavos. Los trabajadores costureros de la ciudad de Buenos Aires.

${ }^{11}$ RIZEK, Cibele et alii. Trabalho e imigração: uma comparação Brasil-Argentina.
} 
de denunciante, se argumenta que estas personas carecen de todo tipo de derechos en nuestro país y que al encontrarse solos nadie hará nada por ellos. Tal ejercicio de violencia puede sostenerse con la complicidad de la policía, tanto federal como provincial, quienes pasarían a "cobrar" por los talleres una "coima" mensual a cambio de impunidad.

Al igual que lo que sucede en BA, los modos de vida/trabajo/vivienda de los "costureiros" bolivianos en SP se caracterizan por su precariedad, en condiciones que se acercan a la esclavitud. En concordancia con el caso porteño, las condiciones de vida de la gran mayoría de inmigrantes bolivianos de SP - mismos que se encuentran indocumentados - están marcadas por una hipertrofia del espacio de sociabilidad, reducido al lugar de vivienda y de trabajo (el taller textil clandestino). Esto favorece la invisibilización de esta población en el espacio público, aumenta su vulnerabilidad frente a la explotación de los talleristas y acota sus posibilidades de dar a conocer su situación de semi esclavitud en pos de revertir la vulneración de sus derechos (a la atención sanitaria, a la educación, etc.). ${ }^{12}$ Es decir que, dentro del taller, se perpetúan

toda una serie de relaciones de dominación, de violencia física y simbólica cotidiana, de presiones y amenazas sutiles (y no tanto) hacia los trabajadores que (...) ante la falta de desconocimiento sobre las formas de trabajo en el país y otro tanto por la falta de opciones y alternativas con las que cuentan para vivir, 'aceptan' tales condiciones laborales de explotación y precariedad. ${ }^{13}$

El relato de "Ro", mujer inmigrante boliviana, ex trabajadora en un TTC de BA, grafica la realidad de estos talleres:

Hacíamos camperas para buenas marcas pero nosotros no sabíamos que eran marcas así grandes no, hacíamos camperas y él nos pagaba uno cincuenta $[\$ 1,50]$ por campera, y teníamos que armar toda la campera entera (...) desde la siete de la mañana a una, dos de la mañana trabajábamos (...) teníamos una hora de almuerzo, para el té, en cuanto acabes de tomar el té tenías que ir a trabajar (...) ahí vivíamos, tenía él la mayoría parejas, tenía piezas precarias y a nosotros nos había dado una pieza precaria donde también tenía máquinas (...) nos tenía bajo llave encerrados, no nos dejaba salir. (Entrevista en profundidad a "Ro").

En consecuencia, es posible enumerar, inicialmente, las siguientes situaciones y factores concretos de riesgo ligados a la emergencia, el contagio,

${ }^{12}$ CYMBALISTA, Renato; XAVIER, lara. A comunidade boliviana em Sao Paulo: definindo padrões de territorialidade.

${ }^{13}$ DOMÍNGUEZ, Carolina. Procesos de identificación y diferenciación en familias y jóvenes bolivianos del AMBA, p. 89. 
la transmisión y el padecer de la TBC en relación al contexto y las condiciones de vivienda, de trabajo y medioambientales frecuentemente presentes en los TTC: la falta de renovación del aire, la forma de la construcción y disposición de las ventanas (poca luz), la acumulación de polvo, la humedad de paredes, suelos y techos, entre otras. Sumado a que el trabajo se realiza en condiciones de hacinamiento, en habitaciones sin ventilación plagadas de retazos de tela, hilos, polvillo en el ambiente y pelusas que producen las máquinas. Y subrayando que la cantidad de horas trabajadas (entre 15 y 18) y las características del mismo provocan en general serios problemas posturales, respiratorios y de la vista; que en el caso de las embarazadas, la intensidad del trabajo, sumada a la escasa y deficiente alimentación ha producido, además de riesgo de vida para la madre y el bebé, anemias crónicas y una baja en las defensas que puede llevar a contraer enfermedades como la TBC. Adicionalmente, vale subrayar que un ambiente mal ventilado, donde las partículas portadoras de los bacilos de Koch pueden circular sin quedar expuestas a los efectos letales de la luz solar, amplifica aún más el riesgo de infección:

apresentando problemas como pouca ventilação ou cadeiras impróprias para a atividade, o que resulta em una série de problemas de saúde aos trabalhadores, além de tuberculose e lesão por esforço repetitivo (como tendinites, artroses, etc.) (...) a exploração dos trabalhadores bolivianos é tão exacerbada que chega a se configurar situação análoga à escravidão, posto que muitos proprietários proíbem os trabalhadores de sair das oficinas, ameaçando entregá-los à Polícia Federal. ${ }^{14}$

\section{Riesgos de infección, contagio, transmisión y desarrollo de la tuberculosis en talleres textiles clandestinos de Buenos Aires y São Paulo}

El taller la enfermó, y así, vencida/en plena juventud, quizás no sabe/de una hermosa esperanza que acaricie/sus largos sufrimientos de incurable (...) Ha tosido de nuevo. El hermanito/Después se ha levantado, y bruscamente/se ha ido, murmurando al alejarse,/con algo de pesar y mucho de asco:/ que la puerca, otra vez escupe sangre. ${ }^{15}$

Cuando hablamos de TBC nos estamos refiriendo a una de las enfermedades infecciosas que, desde la antigüedad, más muertes ha producido y sigue produciendo en el planeta: actualmente, es la segunda causa de mortalidad entre todas las enfermedades infecciosas. En consecuencia, continúa siendo uno de los mayores problemas sanitarios a escala mundial. ${ }^{16}$

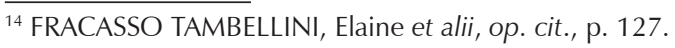

${ }^{15}$ CARRIEGO, Evaristo. Residuo de Fábrica.

${ }^{16}$ WHO. Global tuberculosis report 2012. 
La TBC ha sido considerada como una enfermedad de la "pobreza" desde el momento en que el "mundo subalterno" de los pobres constituye un escondite adecuado para las enfermedades infecciosas, especialmente cuando se trata de situaciones de segregación y marginación social. ${ }^{17}$ Así mismo, constituye en un indicador de la forma en que las desigualdades sociales se traducen en desigualdades en salud..$^{18}$ En tal sentido, el deterioro físico, psicológico y anímico relacionado con la explotación semi esclavista a la cual se somete actualmente a los inmigrantes bolivianos en los TTC de BA y SP plantea una situación similar a la que aconteció durante la primera mitad del siglo XX en Argentina. El extracto del poema citado más arriba, forma parte del amplio registro existente en aquella época en relación a la presencia de la TBC entre las mujeres trabajadoras "(...) como una evidencia de la injusticia social" ${ }^{19} \mathrm{Al}$ igual que en aquel entonces, cuando los trabajadores precarios eran inmigrantes europeos, la TBC puede ser considerada como "la enfermedad emblemática" que incorpora la desigualdad social y cultural existente en la sociedad. ${ }^{20}$ Lo anterior tiene su manifestación en las trayectorias de mujeres inmigrantes bolivianas entrevistadas durante el desarrollo del trabajo de campo de mis investigaciones. ${ }^{21}$ Los distintos casos relevados muestran a mujeres engañadas desde origen por algún familiar o conocido que les promete un trabajo a cambio de un salario en dólares; y que una vez que llegan a destino se encuentran con una realidad que nunca imaginaron previamente. Eso fue lo que le ocurrió a "Ne", quien terminó trabajando en un taller de costura ilegal ubicado en el corazón de una de las más grandes y pobladas villas miserias (favelas) de BA. Además de confirmar la descripción ya expuesta, acerca de la duración y la intensidad de la jornada laboral, las

\footnotetext{
${ }^{17}$ FARMER, Paul. Pathologies of Power. Health, human right, and the new waronthepoor.

${ }^{18}$ GOLDBERG, SILVEIRA, op. cit.

${ }^{19}$ ARMUS, Diego. La ciudad impura: salud, tuberculosis y cultura en Buenos Aires. 1870-1950, p. 119.

${ }^{20}$ FASSIN, Didier. L'espacepolitique de la santé. Essai de généalogie.

${ }^{21}$ Entre ellas, pueden mencionarse las siguientes: “Una aproximación antropológica a la Tuberculosis en inmigrantes bolivianos del Área Metropolitana de Buenos Aires: su relación con los modos de vida y de trabajo", Consejo Nacional de Investigaciones Científicas y Técnicas de la República Argentina, programación 2010-2012. Director: Dr. Alejandro Goldberg; "Las condiciones de trabajo en los talleres textiles de la Ciudad Autónoma de Buenos Aires: factores de riesgo e impacto en la salud/ enfermedad de los trabajadores", Superintendencia de Riesgos del Trabajo, programa de Promoción de la Investigación, Formación y Divulgación sobre Riesgos del Trabajo, Ministerio de Trabajo, Empleo y Seguridad Social de la Nación, período: octubre 2010-septiembre de 2011. Director: Dr. Alejandro Goldberg; "Análisis de la relevancia de los factores socioculturales en el proceso asistencial de pacientes con tuberculosis usuarios del Instituto Vaccarezza-Hospital Muñiz. Un abordaje etnográfico desde la Antropología Médica", Ministerio de Salud de la Nación. Comisión Nacional Salud, Ciencia y Tecnología: Becas "Carrillo - Oñativia", período: 2009-2010. Director: Dr. Alejandro Goldberg; "Itinerarios terapéuticos de inmigrantes bolivianos del Área Metropolitana de Buenos Aires", UBACiencia y Tecnología, programación 2008-2010. Director: Dr. Alejandro Goldberg.
} 
condiciones edilicias del taller, la falta de una alimentación adecuada, los malos tratos por parte del tallerista, etc., "Ne" narró que dormían cuatro personas en una pequeña habitación y que un día comenzó con fuertes dolores de espalda, primero, y de tórax después. Como he podido comprobar de manera recurrente en las narrativas de las entrevistadas, a pesar de las dolencias, malestares y padecimientos sufridos por las trabajadoras, estas continúan desarrollando sus tareas por miedo a las posibles represalias de los dueños del taller. En otros casos más extremos registrados, directamente los/ las trabajadores/as tienen la prohibición de salir del recinto del taller debido a las condiciones de semi-esclavitud en las que se encuentran. "Ne" siguió trabajando hasta que empezó a toser sangre, momento en que su marido, que también trabajaba en el taller, la llevó de urgencia al Hospital Muñiz de la ciudad de BA, donde quedó internada por tres meses con TBC con un estadio avanzado de la enfermedad.

A las situaciones de riesgo concretas en el trabajo se le suman aquellas - muchas de las cuales son las mismas al encontrarse en el recinto del taller ligadas a las condiciones de vivienda; y, adicionalmente, factores vinculados con la presión psicológica a las que son sometidas y el estado emocional en el que se encuentran muchas de estas trabajadoras inmigrantes. Tal es el caso de "Jr", joven boliviana inmersa dentro un proceso migratorio "obligado" por la necesidad de venir a trabajar para pagar deudas asfixiantes en origen. En este sentido, en opinión de "Fr", una de las psicólogas integrantes del equipo de salud del Hospital Muñiz entrevistadas, esto último actuó como factor interactuante y al mismo tiempo determinante en el desarrollo de TBC en esta mujer. De modo que existen también factores socioculturales de peso que impactan en la baja de las defensas de los y las trabajadores/as, pudiendo favorecer el contagio y el desarrollo de esta enfermedad.

Tal como se desprende de lo expuesto para el caso de BA, al aplicar la variable género nos encontramos que en el caso de SP son también las mujeres quienes sufren con mayor intensidad y con peores consecuencias las distintas situaciones de violencia (estructural, física, psicológica) y de riesgo para su salud presentes en el modo de vida/trabajo/vivienda en los TTC. ${ }^{22} \mathrm{Al}$ respecto, vale señalar que las mujeres son más requeridas que los hombres como mano de obra en estas unidades productivas ilegales, ya que, además de la costura, desarrollan otras tareas: cocinar, limpiar, ayudar y aprender el oficio en el recinto del taller (donde, además, viven, en muchos casos con sus hijos y

22 MADI, María C. et alii. Estudo das representaçõessociais sobre gestaçãoemmulheres bolivianas no contexto da atenção básica em saúde na área central da cidade de São Paulo; CARNEIRO JUNIOR, Nivaldo et alii. Migração, exclusão social e serviços de saúde: o caso da população boliviana no centro da cidade de São Paulo. 
su marido que trabaja de costurero), con una sobrecarga laboral intensa y por momentos ininterrumpida. Por lo tanto, se encuentran doblemente expuestas a distintos problemas de salud, entre ellos a la infección y el desarrollo de la TBC.

Por el lado de los profesionales de la salud pública entrevistados como parte del trabajo de campo etnográfico realizado, nos encontramos con que conocen perfectamente las situaciones referidas:

El que no viene infectado desde Bolivia se infecta acá por la comida, la alimentación que lleva, mal nutrición, el trabajo malo... En este momento lo que se ve, lo que prevalece es en los talleres... tiene que ver con las condiciones y medidas de higiene. (Entrevista en profundidad a "F", médica del Hospital Muñiz de BA).

La gente boliviana que vienen de trabajar en un taller todo el día y los tienen hacinados (...) Entonces cómo trabajan ellos, cómo están hacinados, la falta de alimentación y demás, las vitaminas adecuadas para poder mantener... Están sin ventilación adecuada y todo eso, los lleva a enfermarse y cuando se enferma uno de ellos, se contagian todos los que están en los talleres. Entonces vienen unos tras otros a internarse. A veces hay 4 o 5 bolivianos que son del mismo taller. (Entrevista en profundidad a "A", enfermero del Hospital Muñiz de BA).

Si bien la mayor parte de los casos notificados de TBC hasta 2011 inclusive en la ciudad de BA (2.499) seguían correspondiendo a "nativos" argentinos, los casos referidos a inmigrantes bolivianos han mostrado un crecimiento sostenido, fundamentalmente desde 2004 en adelante. Un dato relevante lo constituye el número de casos registrados en el área programática del Hospital Piñero (198 casos por 100.000 habitantes, muy por encima de la tasa global de la ciudad y superando la media nacional argentina), ubicado en el sur-oeste de BA. Esta área concentra los barrios más pobres, abandonados y precarizados de la ciudad, que sufren las consecuencias actuales de desinversión en políticas públicas sociales (educación, salud, vivienda, servicios, medioambiente, trabajo, seguridad) del gobierno local; y dentro de esta área se localizan muchos de los TTC donde trabajan y viven inmigrantes bolivianos con sus familias. ${ }^{23}$

En el caso de SP, los barrios de Belém, Bom Retiro, Brás y Pari, pertenecientes al área más antigua de la ciudad, y con condiciones de vida que se han deteriorado en las últimas décadas generando un empeoramiento general de los indicadores sociales, económicos y de salud, han experimentado en paralelo en los últimos diez años un aumento en la incidencia de la TBC entre inmigrantes bolivianos, invirtiéndose la tendencia de casos de esta enfermedad a favor de los "nativos" brasileños (disminución del 45\%),

${ }^{23}$ GOLDBERG, SILVEIRA, op. cit. 
respecto a los bolivianos (aumento del 250\%). ${ }^{24}$ Un estudio reciente, realizado en ese mismo contexto socio espacial de la ciudad, sugiere que la migración proveniente de países donde la incidencia de la TBC es alta (como el caso de Bolivia) podría llegar a impactar en la incidencia de esta enfermedad a nivel de los indicadores y de la población "nativa". ${ }^{25}$ Dicha afirmación se apoya en investigaciones clínicas llevadas a cabo en países europeos como España, Reino Unido, Alemania y Noruega, las cuales concluyeron que los inmigrantes ocasionalmente se infectan en la sociedad de destino; todo lo cual demostraría que la infección tendría su origen en sus propios países, antes de su llegada a Europa. ${ }^{26}$

Contrariamente a esta perspectiva, en el caso de BA más que asociar TBC con inmigración boliviana en términos de enfermedad "importada" o "cultural", en el sentido de que los bolivianos traen la enfermedad desde su país y se corre el riesgo de que infecten a la población "nativa", el fenómeno se vincula con los modos de vida/vivienda/trabajo que experimentan y sufren estos inmigrantes, tal y como se abordó en este artículo, y con seguridad muy diferentes - con peores condiciones - a los de los inmigrantes que formaron parte de la muestra de los referidos estudios europeos. La hipótesis expuesta para el caso porteño ha sido corroborada a partir de distintos estudios de biología molecular, en los que se demuestra la inexistencia de evidencias científicas de transmisión de cepas extranjeras autóctonas andinas a pacientes argentinos ("nativos") a través de los inmigrantes que llegan enfermos o contraen la enfermedad en BA; pero sí sucede a la inversa: la cepa $M$, dominante en el Área Metropolitana de Buenos Aires (AMBA) comenzó a propagarse entre muchos de los inmigrantes bolivianos que contrajeron la enfermedad en los TTC. ${ }^{27} \mathrm{Si}$ bien los citados estudios realizados en países "desarrollados", a partir de datos epidemiológicos y de genotipificación de ADN, sugieren que la inmigración procedente de zonas hiperendémicas no tiene influencia significativa en el patrón de transmisión de la TBC entre la población "nativa", no obstante lo atribuyen, por un lado, a los contactos limitados entre los

${ }^{24}$ MARTÍNEZ, Vanessa. Equidade em saúde: o caso da tuberculose na comunidade boliviana no município de São Paulo.

${ }^{25}$ MARTíNEZ, Vanessa et alii. Equity in health: tuberculosis in the Bolivian immigrant community of São Paulo, Brazil.

${ }^{26}$ DAHLE, Ulf et alii. Molecular epidemiology of Mycobacterium tuberculosis in Norway; GILBERT, Robert et alii. Theimpact of immigration on tuberculosis rates in the United Kingdom compared with other European countries; DAHLE, UIf et alii. Impact of immigrationonthe molecular epidemiology of Mycobacterium tuberculosis in a low-incidence country; IÑIGO, Jesús et alii. Analysis of changes in recent tuberculosis transmission patterns after a Sharp increase in immigration; BARNIOL, Judith et alii. Transmission dynamics of pulmonary tuberculosis between autochthonous and immigrant sub-populations.

${ }^{27}$ GOLDBERG, SILVEIRA, op. cit. 
diferentes grupos poblacionales de sus países; y, por otro lado, a la eficacia de los programas de detección de casos de TBC en inmigrantes. ${ }^{28}$ Se trata de dos tipos de realidades, en contextos y con situaciones lo suficientemente diversas como para poder extrapolar resultados de unas a otras. Distinto a lo que sucede con los dos casos abordados en este trabajo, en el cual se analizó, en clave comparativa y desde una perspectiva sociosanitaria y regional, la incidencia de la TBC en inmigrantes bolivianos que trabajan y viven en condiciones de extrema precariedad, cercanas a la esclavitud, en TTC ilegales de BA y SP. Del mismo modo, se remarca la importancia de los estudios realizados a través de una perspectiva antropológica-médica del padecimiento para abordar enfermedades infecciosas como la TBC, ya que los enfoques clínicos y epidemiológicos poseen limitaciones propias del método y el procedimiento de recolección de datos (cuantitativos) que utilizan. En este sentido, como parte de la metodología cualitativa adoptada, el enfoque narrativo de la enfermedad permite recuperar al sujeto-paciente ubicándolo en el centro del análisis contextualmente y en el marco de un sistema de relaciones y redes socioculturales. Así, enfatizando en lo holístico, se articulan los niveles micro y macro en el análisis de los problemas de salud, que en el caso de este trabajo intentó aportar a una cierta capacidad explicativa al vincular y comparar dichos procesos con las manifestaciones de morbimortalidad por TBC que presentan los inmigrantes bolivianos que trabajan en TTC en ambas ciudades.

\section{Comentarios finales}

La propuesta de un análisis comparativo, aplicando un enfoque relacional desde una perspectiva sociocultural del fenómeno a escala regional, ha permitido abordar las dimensiones sociosanitarias de una enfermedad como la TBC y su incidencia en un conjunto subalterno específico de la población de las ciudades de BA y SP, cuya cotidianeidad se reproduce en contextos de vulnerabilidad social cercanos a la esclavitud, atravesados por situaciones concretas de riesgo para su salud. Siguiendo este razonamiento, contraer la TBC entre los inmigrantes bolivianos que son explotados ilegalmente en TTC de ambas ciudades, constituye una resultante compleja de sus modos de vida/trabajo/vivienda en estas sociedades, consustanciales con sus procesos migratorios que se dan con frecuencia a través de delitos como la trata, el tráfico de personas y la reducción a la servidumbre.

Debido a las condiciones de semi esclavitud en las que trabajan y viven en los TTC, los sujetos y sus hijos tienen relativamente limitado el derecho a la

${ }^{28}$ MARTÍNEZ et alii, op. cit. 
atención sanitaria de sus problemas de salud; en este caso, a la consulta y a un tratamiento eficaz por TBC en el sistema público (más en el caso porteño que en el paulista). En relación a esto último, la acción de los agentes comunitarios de salud en SP ingresando a las "oficinas" de costura e interrogando a los trabajadores a fin de rastrear una posible sintomatología asociada a la TBC, no está contemplada en el caso de BA, donde resulta imposible - además de inseguro - ingresar al recinto de un taller textil clandestino. Adicionalmente, la formación de agentes comunitarios de salud pertenecientes al colectivo inmigrante boliviano como sucede en el caso de Brasil, constituiría un avance en la prevención de nuevos contagios y en la eficacia del tratamiento.

Por último, se subraya que la posibilidad de terminar con las situaciones de explotación referidas, disminuir así en parte los riesgos de infección, transmisión y desarrollo de la TBC entre los sujetos de los casos abordados, y apostar al éxito del tratamiento, dependería principalmente del cumplimiento de los marcos constitucionales y las leyes de inmigración vigentes en Argentina y Brasil, lo cual implica legalizar los talleres y regularizar a los trabajadores, por un lado; y, simultáneamente, garantizar los derechos humanos y sociales fundamentales de estas y todas las personas que habitan estos países, incluyendo los de acceso universal e irrestricto a la atención sanitaria pública gratuita de calidad, y a un trabajo y una vivienda digna.

\section{Referencias bibliográficas}

ARMUS, Diego. La ciudad impura: salud, tuberculosis y cultura en Buenos Aires. 1870-1950. Buenos Aires: Edhasa, 2007.

BALÁN, Jorge. La economía doméstica y las diferencias entre los sexos en las migraciones internacionales: un estudio sobre el caso de los bolivianos en la Argentina. Estudios Migratorios Latinoamericanos, año V, n. 15-16, 1990, p. 269-294.

BARNIOL, Judith et alii. Transmission dynamics of pulmonary tuberculosis between autochthonous and immigrant sub-populations. Infectious Diseases, v. 9, december/2009, p. 197.

BENENCIA, Roberto. Apéndice: La inmigración limítrofe. In DEVOTO, Fernando. Historia de la inmigración en la Argentina. Buenos Aires: Sudamericana, 2003.

BUECHLER, Simone. Sweatinglt in the Brazilian Garment Industry: Korean and Bolivian Immigrants and Global Economic Forces in São Paulo. Latin American Perspectives, v. 31, n. 3, East Asian Migrationto Latin America, May/2004, p. 99-119.

CARNEIRO JUNIOR, Nivaldo et alii. Migração, exclusão social e serviços de saúde: o caso da população boliviana no centro da cidade de São Paulo. Boletim do Instituto de Saúde, São Paulo, v. 13, n. 3, outubro/2011.

CARRIEGO, Evaristo. Residuo de Fábrica. In IDEM. Poesías completas. Buenos Aires: EUDEBA, 1968 [1926]. 
CYMBALISTA, Renato; XAVIER, lara. A comunidade boliviana em Sao Paulo: definindo padrões de territorialidade. Cadernos da Metrópole, n. 17, I/2007, p. 119-133.

DAHLE, Ulf et alii. Molecular epidemiology of Mycobacterium tuberculosis in Norway. Journal of Clinical Microbiology, v. 39, n. 5, May/2001, p. 1802-1807.

. Impact of immigration on the molecular epidemiology of Mycobacterium tuberculosis in a low-incidence country. American Journal of Respiratory Critical Care Medicine, v. 176, August/2007, p. 930-935.

DA SILVA, Sidney. Bolivianos em São Paulo: entre o sonho e a realidade. Estudos Avançados, v. 20, n.57, may-aug/2006, p. 157-170.

DOMÍNGUEZ, Carolina. Procesos de identificación y diferenciación en familias y jóvenes bolivianos del AMBA. Tesis de Licenciatura en Antropología Social, Universidad de Buenos Aires, 2010.

FARMER, Paul. Pathologies of Power. Health, human right, and the new waronthepoor. Berkeley: U. of California Press, 2003.

FASSIN, Didier. L'espace politique de la santé. Essai de généalogie. París: PressesUniversitaires de France, 1996.

FRACASSO TAMBELLINI, Elaine et alii. Inclusão de imigrantes bolivianos na cidade de São Paulo: algumas estratégias e políticas locais. In SILVEIRA, Cássio et alii (org.). Projeto inclusão social urbana: nós do centro. Metodologia de pesquisa e de ação para inclusão social de grupos em situação de vulnerabilidade no centro da cidade de Sao Paulo. São Paulo: Fundaçao Arnaldo Vieira de Carvalho, 2009.

FREIRE DA SILVA, Carlos. Precisa-se: bolivianos na industria de confecções em São Paulo. Travessia, ano XXII, n. 63, jan-abr/2009, p. 5-11.

GILBERT, Robert et alii. The impact of immigration on tuberculosis rates in the United Kingdom compared with other European countries. Int J TubercLung Dis, v. 13, n. 5, May/2009, p. 645-51.

GOLDBERG, Alejandro. Análisis de la relevancia de los factores socioculturales en el proceso asistencial de pacientes con tuberculosis, usuarios del Instituto VaccarezzaHospital Muñiz. Un abordaje etnográfico desde la Antropología Médica. Revista Argentina de Salud Pública, v. 1, n. 5, diciembre/2010, p. 13-21.

. Servitude and slave trade: the case of Bolivian immigrants who work in clandestine textile workshops of the Buenos Aires MetropolitanArea. Miradas en Movimiento, v. Especial "Naturallylmmigrants", enero/2012, p.188-202.

. Tuberculosis y vulnerabilidad social. Una investigación etnográfica sobre inmigrantes bolivianos que trabajan y viven en talleres textiles clandestinos del Área Metropolitana de Buenos Aires. Saarbrücken: Editorial Académica EspañolaLAP LAMBERT Academic Publishing, 2012.

GOLDBERG, Alejandro; SILVEIRA, Cássio. Modos de vida, condiciones de acceso a la salud pública y procesos de atención en inmigrantes bolivianos de Buenos Aires y São Paulo: una indagación comparativa. Saúde e Sociedade (en evaluación).

GRIMSON, Alejandro. Relatos de la diferencia y la igualdad. Los bolivianos en Buenos Aires. Buenos Aires: Eudeba, 1999. 
IÑIGO Jesús et alii. Analysis of changes in recent tuberculosis transmission patterns after a Sharp increase in immigration. Journal of Clinical Microbiology, v. 45, n. 1, jan/2007, p. 63-69.

LIEUTIER, Ariel. Esclavos. Los trabajadores costureros de la ciudad de Buenos Aires. Buenos Aires: Retórica Ediciones, 2010.

MADI, María C. et alii. Estudo das representações sociais sobre gestação em mulheres bolivianas no contexto da atenção básica em saúde na área central da cidade de São Paulo. Saúde e Sociedade, v. 18, sup. 2, apr-jun/2009, p. 67-71.

MARTÍNEZ, Vanessa. Equidade em saúde: o caso da tuberculose na comunidade boliviana no município de São Paulo. Tesis de Maestría, Faculdade de Saúde Pública, Universidade de São Paulo, 2010.

MARTÍNEZ, Vanessa et alii. Equity in health: tuberculosis in the Bolivian immigrant community of São Paulo, Brazil. Tropical Medicine \& International Health, v. 17, November/2012, p. 1417-1424.

RIZEK, Cibele et alii. Trabalho e imigração: uma comparação Brasil-Argentina. Lua Nova, n. 79, 2010, p. 111-142.

SASSONE, Susana; DE MARCO, Graciela. Problemáticas territoriales de la inmigración limítrofe. In DE MARCO, Graciela et alii. Extranjeros en la Argentina. Pasado, presente y futuro. Buenos Aires: Geodemos, PRIGEO-CONICET, 1994.

SOUCHAUD, Sylvain; BAENINGER, Rosana. Collas e cambas do outro lado da fronteira: aspectos da distribuição diferenciada da imigração boliviana em Corumbá, Mato Grosso do Sul. Rev. Bras. Estud. Popul., v. 25, n. 2, july-dec/2008, p. 271-286.

WHO. Global tuberculosis report 2012. Ginebra: WHO, 2012.

\section{Abstract}

\section{A comparative approach of the impact of tuberculosis among bolivian immigrants of Buenos Aires and São Paulo}

This paper presents an exploratory comparative approach of some dimensions of analysis among the incidence of tuberculosis in Bolivian immigrants of Buenos Aires and São Paulo cities, based on a sociosanitary and regional perspective. In both contexts, tuberculosis is linked to migrant's life styles and precarious working conditions in clandestine textile workshops. In addition, their lack of documentation, their problems to access to public health care and the social suffering they experience, jointly deepen Bolivian immigrants social vulnerability. Infection risks are thus increased.

Keywords: Bolivian immigrants; Clandestine textile workshops; Social vulnerability; Tuberculosis.

Recebido para publicação em 10/03/2013.

Aceito para publicação em 13/05/2013. Received for publication in March, 10 ${ }^{\text {th }}, 2013$. Accepted for publication in May, 13 ${ }^{\text {th }}, 2013$. 